\title{
Tuberculosis following renal transplantation in England, Wales and Northern Ireland: a national registry-based cohort study
}

\section{To the Editor:}

Increased tuberculosis (TB) disease risk has consistently been observed among solid organ transplant recipients $[1,2]$. This may result from post-transplant immunosuppressive therapy, although underlying disease processes leading to transplant (including chronic kidney disease) are also associated with increased risk [3]. TB among transplant recipients is associated with increased risk of drug toxicity, drugdrug interactions, graft failure and mortality [4], and can lead to transmission to other vulnerable hospitalised patients. Consequently, global guidelines recommend systematic screening of patients undergoing solid organ transplant for latent tuberculosis infection (LTBI) [3].

Renal transplants represent $70 \%$ of all transplants performed annually in the UK [5]. Few studies have examined the incidence of TB post-renal transplant in low TB incidence settings; these have reported incidence rates 17 - to 30 -fold higher than in the general population $[1,2,4,6]$. However, no studies have been conducted in the UK, and none have calculated ethnicity-stratified rates. The latter is important as minority ethnic groups are over-represented among renal transplant recipients, and are also at increased risk of TB disease.

In England, Wales and Northern Ireland, the Enhanced TB Surveillance (ETS) system contains data on all TB notifications, while the UK Transplant Registry, held by NHS Blood and Transplant (NHSBT), provides national level data on transplant recipients. This includes detailed recipient characteristics, transplant information and annual post-transplant follow-up data on graft and patient survival. We aimed to utilise these datasets to investigate ethnicity-stratified TB incidence rates among renal transplant recipients in the $\mathrm{UK}$, and to examine risk factors for incident TB, to inform future testing and treatment policy.

Data on renal transplant recipients in England, Wales and Northern Ireland from the UK Transplant Registry were deterministically matched with the ETS using unique NHS numbers in order to identify transplant recipients who were notified with TB disease during the study period (January 2000 to December 2013).

Renal transplant recipients entered the study cohort on the date of their first renal transplant during the study period. Follow-up was censored on the earliest date of: first TB diagnosis; death; or 31 December 2013 (the date of data extraction). Notified TB cases included culture-confirmed TB or clinically diagnosed with radiological or histological evidence of $\mathrm{TB}$, where a clinician had prescribed treatment with a full course of anti-TB treatment. The primary outcome measure was the crude post-transplant TB incidence rate, stratified by ethnicity (defined as either white, Asian (Indian, Pakistani or Bangladeshi), or "other"). Crude incidence rates were calculated per 100000 person years, with 95\% confidence intervals. Incidence rates were calculated during and after the first year post-transplant, since we hypothesised that TB risk would be highest in the initial months following transplantation, when the burden of immunosuppression is highest [2].

@ERSpublications

Among a nationwide cohort of renal transplant recipients $(n=30433)$, TB incidence was highest in the first year post-transplant. Asian ethnicity and CMV seropositivity were independent risk factors for post-transplant TB http://bit.ly/2YSVGt2

Cite this article as: Gupta RK, Rosenberg G, Eriksen J, et al. Tuberculosis following renal transplantation in England, Wales and Northern Ireland: a national registry-based cohort study. Eur Respir J 2019; 54: 1802245 [https://doi.org/10.1183/13993003.02245-2018]. 
Risk factors for incident TB among the transplant cohort were investigated using Cox regression with death as a competing risk, in view of the high mortality among the cohort. Exposure variables included recipient age at transplant, gender, ethnicity, cytomegalovirus (CMV) seropositivity, and co-infection with HIV, hepatitis B and hepatitis C. These variables are routinely collected by the UK Transplant Registry. Variables with $\mathrm{p}<0.2$ in univariable analysis were included in the final multivariable model.

Public Health England has authority under the Health and Social Care Act 2012 to hold and analyse national surveillance data for public health and research purposes. All patient data were fully anonymised prior to analysis.

A total of 30433 unique renal transplant recipients were included. Median age at entry to the cohort was 44 years (interquartile range (IQR) 36-55 years), and a majority (18723/30 433; 61.5\%) of individuals were male. A total of 24951/30433 (82.0\%), 3055/30433 (10.0\%) and 2189/30433 (7.2\%) were of white, Asian and "other" ethnicity, respectively. The majority of patients (28305/30433; 93.0\%) had single renal transplants only. CMV seropositivity was common, affecting 12658/30433 (41.6\%) of the cohort; this was more common among patients of Asian and "other" ethnicity, compared to the white ethnic group (9291/ 24951 (37.2\%) in white group versus 1899/3055 (62.2\%) in Asians and 1371/2189 (62.6\%) in "other" ethnic group). Conversely, only $147 / 30433$ (0.5\%), 244/30433 (0.8\%) and 415/30433 (1.4\%) of patients were known to be co-infected with HIV, hepatitis C virus and hepatitis B virus, respectively. A total of $3299 / 30433$ (10.8\%) transplants were done due to underlying diabetic renal disease. Median follow-up was 5.1 years (IQR 2.2-8.7 years).

Over 172421 person-years of follow-up, 53/30433 (0.17\%) recipients were notified with incident TB, yielding an overall rate of 30.7/100000 person-years (median time to incident TB among 53 cases was 1.4 years (IQR 0.6-4.0)). During follow-up, 3486/30433 (11.5\%) individuals died (median time to death 3.6 years (IQR 0.9-6.7)).

Among all ethnic groups, crude TB incidence rates were markedly higher in the first year post-transplant than thereafter (30.0 versus 11.6/100000 person-years in white ethnic group; 289.8 versus 125.5/100 000 person-years in Asian ethnic group; 199.0 versus 33.3/100 000 person-years in "other" ethnic group).

In the multivariable competing risks regression analysis (table 1), Asian ethnicity (compared to white) was a strong risk factor for developing incident TB (sub-distribution hazard ratio (sHR) 11.0, 95\% CI 5.2822.91; $\mathrm{p}<0.001)$. CMV seropositivity was also independently associated with increased incident TB risk (sHR 2.42, 95\% CI 1.03-5.68; $\mathrm{p}=0.04$ ).

TABLE 1 Results of univariable and multivariable $(n=24014)$ competing risks Cox regression analysis of risk factors for incident tuberculosis among renal transplant cohort

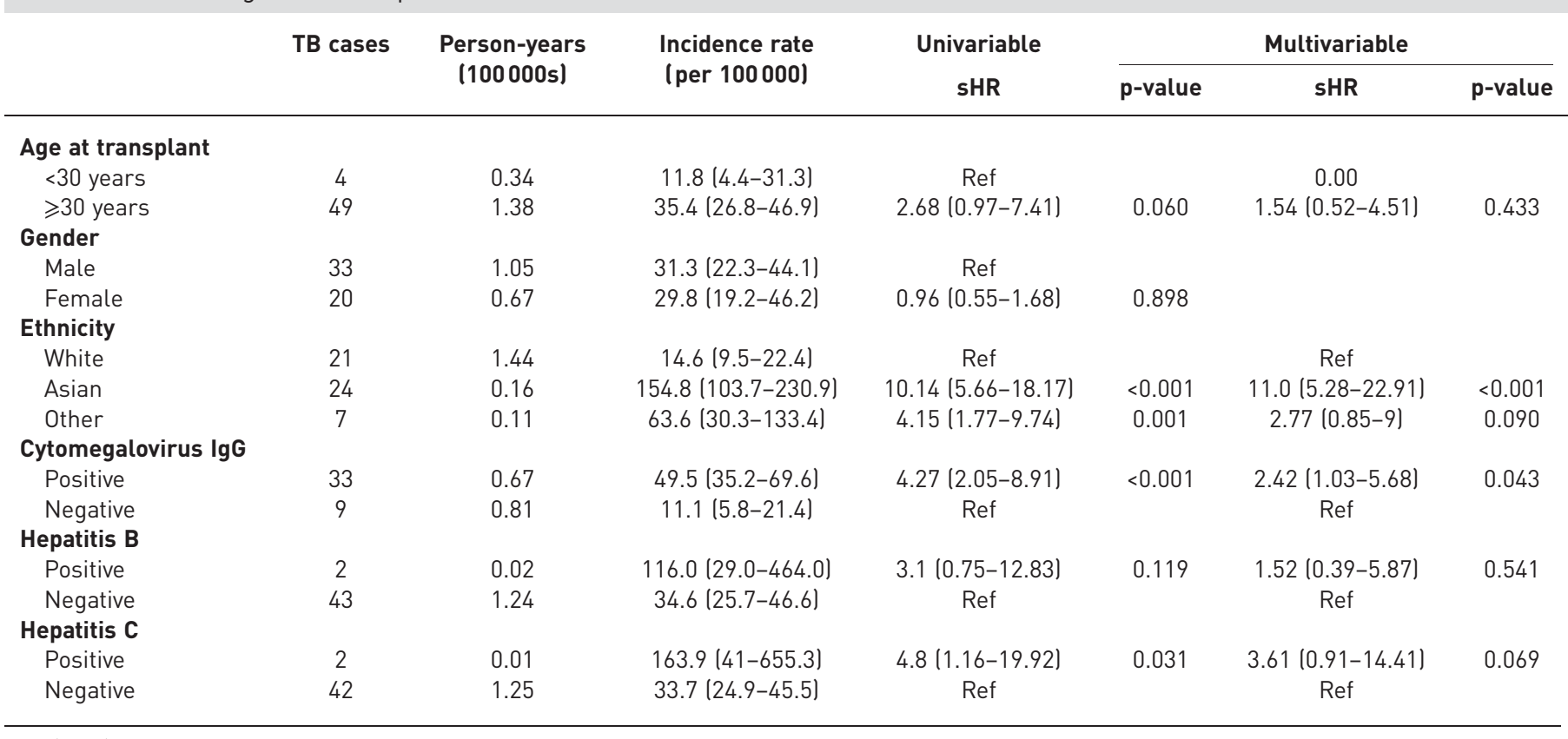

Ss (sHR) Data are presented with $95 \%$ confidence intervals. sHR: subdistribution hazard ratio. 
Of the 53 incident TB cases, 24 (45\%) cases had pulmonary involvement, with the remainder notified as exclusively extra-pulmonary. Only one patient was notified with genitourinary TB.

In this large population-based study examining incidence of TB post-renal transplantation, TB incidence varied markedly by recipient ethnicity, with highest incidence rates among Asian recipients. Among all ethnic groups, TB incidence was considerably higher in the first year post-transplant, suggesting that TB risk is associated with the initiation of post-transplant immunosuppression. The early onset of incident TB events post-transplant suggests that they are likely due to reactivation of latent infection, and therefore supports interventions such as provision of systematic screening for LTBI among patients of Asian and other non-white ethnicity being prepared for renal transplantation [3, 7]. This recommendation is reinforced by the previously observed severe sequelae of incident TB following renal transplant, including a higher risk of graft failure and mortality [4]. Among white transplant recipients, the overall post-transplant TB incidence is markedly lower. An individual risk assessment which combines age and prior TB exposure could inform latent TB testing and treatment decisions in this group.

CMV seropositivity was independently associated with incident TB among transplant recipients. This finding is consistent with data describing increased TB risk associated with CMV, which has led to the hypothesis that CMV co-infection may expedite progression from LTBI to TB disease via T-cell activation $[8,9]$. Further cohort studies and basic science research are needed to elucidate whether there is a causal association between $\mathrm{CMV}$ and $\mathrm{TB}$, and to rule out residual confounding by ethnicity, socioeconomic status or immune susceptibility as alternative explanations.

Strengths of this study include the comprehensive, nationwide study cohort over a 14-year period. Data completeness for key variables was also very high $(99.2 \%$ for ethnicity; $82-89.1 \%$ for viral co-infection screening). A weakness is that no data were available on previous TB disease or LTBI screening pre-transplant, or the provision of TB preventative therapy (which is heterogeneous among transplant centres in the UK [7, 10]). If a large proportion of transplant recipients received prior TB or LTBI therapy, this may have reduced our observed TB incidence rates. Second, our data linkage procedure may have failed to identify some incident TB cases, since NHS numbers were available for approximately $70 \%$ of ETS notifications during the study period, while some transplant patients may also have emigrated during follow-up. The calculated post-transplant incidence rates should therefore be considered as minimum estimates. However, the effect of incomplete linkage is likely non-differential and therefore limited our ability to detect associations rather than creating false associations. Further, NHS number is more likely to be available in ETS for transplant recipients (who are well engaged in NHS care), which would therefore reduce our risk of missing post-transplant TB cases. Finally, available data on country of birth were incomplete, and therefore not included in the analysis.

In summary, we have demonstrated that post-transplant TB incidence is markedly higher among Asian and "other" ethnic groups than the white ethnic group. Ethnicity-stratified TB incidence rates are elevated during the first year post-renal transplant among all ethnic groups. These data support UK guidance recommending systematic LTBI screening among patients being prepared for transplant who are thought to be at high risk of TB. In addition to Asian ethnicity, CMV seropositivity was an independent risk factor for incident TB. The mechanism for this association remains unclear, and requires further investigation.

Rishi K. Gupta $\oplus^{1}$, Gabriel Rosenberg ${ }^{2}$, Jaran Eriksen $\oplus^{2,3}$, Marc Lipman ${ }^{4,5}$, Charlotte Jackson ${ }^{1}$, Anjana Roy ${ }^{6}$, Dominik Zenner ${ }^{1}$, Colette Smith ${ }^{1}$, Mark Harber ${ }^{5}$, Colin Campbell ${ }^{1,6}$ and Ibrahim Abubakar ${ }^{1}$

${ }^{1}$ Institute for Global Health, University College London, London, UK. ${ }^{2}$ Dept of Public Health Sciences, Karolinska Institutet, Stockholm, Sweden. ${ }^{3}$ Dept of Laboratory Medicine (Division of Clinical Pharmacology), Karolinska Institutet, Stockholm, Sweden. ${ }^{4}$ UCL-TB and UCL Respiratory, University College London, London, UK. ${ }^{5}$ Royal Free London NHS Foundation Trust, London, UK. ${ }^{6}$ Public Health England, TB Unit, Colindale, London, UK.

Correspondence: Rishi K. Gupta, Institute for Global Health, 4th Floor Mortimer Market Centre, University College London, WC1E 6JB, UK. E-mail: r.gupta@ucl.ac.uk

Received: 28 Nov 2018 | Accepted after revision: 12 June 2019

Acknowledgements: We are extremely grateful to the NHS Blood and Transplant team and Public Health England TB unit for maintaining and providing the linked data used in this study, along with the transplant centres and TB services who contributed the original data.

Author contributions: A. Roy and I. Abubakar conceived the study and co-ordinated the data linkage. R.K. Gupta and G. Rosenberg performed the analysis, supported by C. Smith, J. Eriksen and C. Jackson. M. Lipman, M. Harber, C. Campbell and D. Zenner provided clinical and policy expertise and insight. R.K. Gupta and G. Rosenberg wrote the manuscript; the final version was critically reviewed and approved by all authors.

Support statement: This work was supported by the National Institute for Health Research (DRF-2018-11-ST2-004 to R.K. Gupta; SRF-2011-04-001 and NF-SI-0616-10037 to I. Abubakar). This paper presents independent research supported by the NIHR. The views expressed are those of the author(s) and not necessarily those of the NHS, the NIHR 
or the Department of Health and Social Care. Funding information for this article has been deposited with the Crossref Funder Registry.

Conflict of interest: R.K. Gupta has nothing to disclose. G. Rosenberg has nothing to disclose. J. Eriksen has nothing to disclose. M. Lipman has nothing to disclose. C. Jackson has nothing to disclose. A. Roy has nothing to disclose. D. Zenner has nothing to disclose. C. Smith reports personal fees for preparation of educational materials from Gilead Sciences and ViiV Healthcare, outside the submitted work. M. Harber has nothing to disclose. C. Campbell has nothing to disclose. I. Abubakar has nothing to disclose.

\section{References}

1 Torre-Cisneros J, Doblas A, Aguado JM, et al. Tuberculosis after solid organ transplant: incidence, risk factors, and clinical characteristics in the RESITRA (Spanish Network of Infection in Transplantation) cohort. Clin Infect Dis 2009; 48: 1657-1665.

2 Singh N, Paterson DL. Mycobacterium tuberculosis infection in solid-organ transplant recipients: impact and implications for management. Clin Infect Dis 1998; 27: 1266-1277.

3 World Health Organization. Latent TB Infection: Updated and Consolidated Guidelines for Programmatic Management. Geneva, World Health Organization, 2018. Available from: www.who.int/tb/publications/2018/ latent-tuberculosis-infection/en/

4 Klote MM, Agodoa LY, Abbott K. Mycobacterium tuberculosis infection incidence in hospitalized renal transplant patients in the United States, 1998-2000. Am J Transplant 2004; 4: 1523-1528.

5 NHS Blood and Transplant. Overview of Organ Donation and Transplantation. https://nhsbtdbe.blob.core. windows.net/umbraco-assets/1819/section-2-overview-of-organ-donation-and-transplantation.pdf

6 Rafiei N, Williams J, Mulley WR, et al. Mycobacterium tuberculosis: active disease and latent infection in a renal transplant cohort. Nephrology 2019; 24: 569-574.

7 British Thoracic Society Standards of Care Committee and Joint Tuberculosis Committee, Milburn H, Ashman N, et al. Guidelines for the prevention and management of Mycobacterium tuberculosis infection and disease in adult patients with chronic kidney disease. Thorax 2010; 65: 557-570.

8 Cobelens F, Nagelkerke N, Fletcher H. The convergent epidemiology of tuberculosis and human cytomegalovirus infection. F1000Research 2018; 7: 280.

9 Muller J, Matsumiya M, Snowden MA, et al. Cytomegalovirus infection is a risk factor for TB disease in infants. bioRxiv 2017; preprint [https://doi.org/10.1101/222646].

10 Maynard-Smith L, Fernando B, Hopkins S, et al. Managing latent tuberculosis in UK renal transplant units: how does practice compare with published guidance? Clin Med (Northfield IL) 2014; 14: 26-29.

The content of this work is copyright of the authors or their employers. Design and branding are copyright 